\title{
Elderly care as one of the important government policy agenda
}

Calvin PW Cheng *, FHKCPsych, FHKAM (Psychiatry)

Department of Psychiatry, The University of Hong Kong, Pokfulam, Hong Kong

* Corresponding author: chengpsy@hku.hk

Hong Kong Med J 2018;24:442-3

DOI: $10.12809 / \mathrm{hkmj} 185084$

According to the latest government population projections, more than a third of the population will be aged $\geq 65$ years by 2046 , with the number of adults aged $\geq 65$ expected to increase from 1190000 in 2016 to 2510000 in 2046 . The soaring elderly population will inevitably put extra strain on our overly stretched health care system, with dementia management being one of our biggest challenges.

More than $8.5 \%$ of community-dwelling older adults suffer from mild cognitive impairment (MCI) and $8.9 \%$ suffer from mild dementia, according to the latest epidemiological study of dementia in Hong Kong. ${ }^{1}$ Dementia is not restricted to only cognitive impairment and functional decline; there is a wide range of behavioural and psychological symptoms of dementia (BPSD), including delusion, disinhibition, violence, irritability, and yelling. These symptoms are distressing not only to the patients themselves, but also to their caregivers. Moreover, the presence of BPSD is also linked to a higher mortality rate, ${ }^{2}$ greater medical expenditure, and earlier institutionalisation. ${ }^{3}$

Early and accurate diagnosis, prevention, and continuous integrated care are vital in across all stages of dementia management. ${ }^{4}$ However, delayed diagnosis of dementia is often seen in Hong Kong, ${ }^{5}$ preventing patients with BPSD from receiving beneficial treatment at an earlier stage. Even when dementia is detected early, neuroimaging investigations should be made to correctly identify the underlying pathology, such as Alzheimer's disease, vascular dementia, dementia with Lewy bodies, or frontotemporal dementia. ${ }^{6}$

Chronic illnesses including diabetes mellitus, hypertension, and depression are closely related to dementia. ${ }^{4}$ Thus, optimisation of these chronic illnesses may reduce the risk and impact of dementia. Furthermore, poor monitoring of private residential care homes for the elderly, along with nonstandardised care, may aggravate risks of infection ${ }^{7}$ and falls, both of which are commonly seen in residents of such facilities. Fall episodes often lead to hip fractures, which are closely linked to sarcopenia ${ }^{8}$ and excess mortality ${ }^{9}$.

The government has repeatedly emphasised on the importance of elderly care in recent years, with one of the key approaches being 'ageing in place' (ie, prioritising care for elderly patients within the community rather than institutionalisation). In particular, the 'money-following-the-user' approach has been adopted, which involves community care service vouchers that can be used to improve the medical care of community-dwelling older adults. Despite these efforts, the existing community service remains inadequate, as indicated by long waiting times for public health care services and the limited scope for the scheme to help with certain conditions such as MCI or mild dementia. Those with more severe dementia or significant BPSD often receive little support. This results in long waiting lists for subsidised residential care homes and creates a further burden on the public health care services.

It is vital to provide a more comprehensive care system in the future, whether as a community, or within institutions or hospitals. At the community level, a continuous and integrated health care system should be available, with services tailored to each individuals needs. For instance, an advisory team including a case manager could closely monitor a patient's medical and social needs. At the institutional level, a more effective monitoring system, better staff training, and increased hiring of experienced care workers from overseas are deemed crucial in improving the quality of care provided by residential care homes for the elderly. Moreover, further land reserves should also be prioritised for creating more residential care facilities to address the current lack of capacity in these institutional services. Within hospitals, a cross-discipline approach should be encouraged. More active cooperation is expected between geriatricians and psychogeriatricians in dementia management, with less reliance on the existing passive referral system, given the diversity of dementia profiles. Outreach services could also be expanded across community and institutional levels as a potential solution to lower the hospitalisation rate.

Given the rapid growth of the ageing population in Hong Kong, there are great challenges ahead in the provision of elderly care. Despite existing efforts by the government, the surging demand for elderly care still outpaces the limited supply across the community, institutional, and hospital levels. Government policy should prioritise 
allocating additional resources to elderly care, in order to address inadequacies in the existing elderly care system in Hong Kong.

\section{Declaration}

The author has disclosed no conflicts of interest.

\section{References}

1. Lam LC, Tam CW, Lui VW, et al. Prevalence of very mild and mild dementia in community-dwelling older Chinese people in Hong Kong. Int Psychogeriatr 2008;20:135-48.

2. Tun SM, Murman DL, Long HL, Colenda CC, von Eye A. Predictive validity of neuropsychiatric subgroups on nursing home placement and survival in patients with Alzheimer disease. Am J Geriatr Psychiatry 2007;15:314-27.

3. Chan DC, Kasper JD, Black BS, Rabins PV. Presence of behavioral and psychological symptoms predicts nursing home placement in community-dwelling elders with cognitive impairment in univariate but not multivariate analysis. J Gerontol A Biol Sci Med Sci 2003;58:548-54.

4. Yee A, Tsui NB, Chang YN, et al. Alzheimer's disease: insights for risk evaluation and prevention in the Chinese population and the need for a comprehensive programme in Hong Kong/China. Hong Kong Med J 2018;24:492500 .

5. Shea YF, Chu LW, Lee SC, Chan AO. The first case series of Chinese patients in Hong Kong with familial Alzheimer's disease compared with those with biomarker-confirmed sporadic late-onset Alzheimer's disease. Hong Kong Med J 2017;23:579-85.

6. Shea YF, Ha J, Lee SC, Chu LW. Impact of ${ }^{18}$ FDG PET and ${ }^{11} \mathrm{C}$-PIB PET brain imaging on the diagnosis of Alzheimer's disease and other dementias in a regional memory clinic in Hong Kong. Hong Kong Med J 2016;22:327-33.

7. Choy CS, Chen H, Yau CS, Hsu EK, Chik NY, Wong AT. Prevalence of infections among residents of Residential Care Homes for the Elderly in Hong Kong. Hong Kong Med J 2016;22:347-55.

8. Ho AW, Lee MM, Chan EW, et al. Prevalence of presarcopenia and sarcopenia in Hong Kong Chinese geriatric patients with hip fracture and its correlation with different factors. Hong Kong Med J 2016;22:23-9.

9. Man LP, Ho AW, Wong SH. Excess mortality for operated geriatric hip fracture in Hong Kong. Hong Kong Med J 2016;22:6-10 